\title{
MEX-3 interacting proteins link cell polarity to asymmetric gene expression in
}

\section{Caenorhabditis elegans}

\author{
Nancy N. Huang ${ }^{1}$, Darcy E. Mootz ${ }^{1}$, Albertha J. M. Walhout ${ }^{2}$, Marc Vidal ${ }^{2}$ and Craig P. Hunter,* \\ ${ }^{1}$ Department of Molecular and Cellular Biology, Harvard University, Cambridge, MA 02138, USA \\ 2Dana-Farber Cancer Institute and Department of Genetics, Harvard Medical School, Boston, MA 02115, USA \\ *Author for correspondence (e-mail: hunter@mcb.harvard.edu) \\ Accepted 9 November 2001
}

\section{SUMMARY}

The KH domain protein MEX-3 is central to the temporal and spatial control of PAL-1 expression in the $C$. elegans early embryo. PAL-1 is a Caudal-like homeodomain protein that is required to specify the fate of posterior blastomeres. While pal-1 mRNA is present throughout the oocyte and early embryo, PAL-1 protein is expressed only in posterior blastomeres, starting at the four-cell stage. To better understand how PAL-1 expression is regulated temporally and spatially, we have identified MEX-3 interacting proteins (MIPs) and characterized in detail two that are required for the patterning of PAL-1 expression. RNA interference of MEX-6, a CCCH zinc-finger protein, or SPN-4, an RNA recognition motif protein, causes PAL1 to be expressed in all four blastomeres starting at the four-cell stage. Genetic analysis of the interactions between these mip genes and the par genes, which provide polarity information in the early embryo, defines convergent genetic pathways that regulate $\mathrm{MEX}-3$ stability and activity to control the spatial pattern of PAL-1 expression. These experiments suggest that par-1 and par-4 affect distinct processes. par- 1 is required for many aspects of embryonic polarity, including the restriction of MEX-3 and MEX-6 activity to the anterior blastomeres. We find that PAL-1 is not expressed in par-1 mutants, because MEX-3 and MEX6 remain active in the posterior blastomeres. The role of par-4 is less well understood. Our analysis suggests that par-4 is required to inactivate MEX-3 at the four-cell stage. Thus, PAL-1 is not expressed in par-4 mutants because MEX-3 remains active in all blastomeres. We propose that MEX-6 and SPN-4 act with MEX-3 to translate the temporal and spatial information provided by the early acting par genes into the asymmetric expression of the cell fate determinant PAL-1.

Key words: Caenorhabditis elegans, Pattern formation, Polarity, RNA-binding protein, Translational control

\section{INTRODUCTION}

A central question in developmental biology is how cells first acquire polarity and then use that polarity to produce daughter cells with distinct patterns of gene expression. In C. elegans, the anteroposterior polarity of the zygote is determined by the position of the sperm pronucleus (Goldstein and Hird, 1996), which organizes a cortical and cytoplasmic rearrangement that leads to the asymmetric distribution of maternally supplied mRNAs and proteins before first cleavage (reviewed by Kemphues and Strome, 1997). Two broad classes of maternally expressed patterning genes have been identified in the $C$. elegans embryo: early acting polarity genes, which are required to establish or maintain embryonic polarity, and cell fate determinants, which act later to direct lineage-specific patterns of development (reviewed by Bowerman, 1998). Defects in polarity genes such as the par genes (partitioning defective) result in early and extensive polarity defects, including loss of asymmetry in cell size, cell cycle time, spindle orientation and distribution of cell fate determinants. Identified cell fate determinants include transcription factors and transmembrane receptors that are asymmetrically distributed among early blastomeres and are required to direct the differentiation of blastomere-specific cell lineages.

While it is known that par gene activity is required to polarize the zygote and enable the proper segregation of cell fate determinants, the mechanisms of action are poorly understood. For example, PAR-1 and PAR-4 are cortically localized serine/threonine kinases; however, none of their phosphorylation targets is known (Guo and Kemphues, 1995; Watts et al., 2000). Similarly, par-3 and par-6 encode conserved PDZ domain proteins that apparently form a protein complex localized to the anterior cortex (Etemad-Moghadam et al., 1995; Hung and Kemphues, 1999; Joberty et al., 2000; Lin et al., 2000). However, the mechanism by which this PAR3/PAR-6 complex confers polarity information to downstream targets is largely unknown. We are interested in understanding how the PAR proteins direct the asymmetric distribution of maternally supplied cell fate determinants.

Considering that maternal factors act to define asymmetries before zygotic transcription begins, the par-dependent distribution of maternal gene activities must be regulated by a variety of post-transcriptional mechanisms, including differential stability of RNA or protein, asymmetric RNA or 
protein localization, and control of mRNA translation. Based on the pleiotropy of the par polarity phenotypes, it is likely that the PAR proteins indirectly regulate the distribution of maternal determinants through intermediate regulators. One candidate intermediate regulator is the RNA-binding protein MEX-3, which regulates the asymmetric expression of maternally encoded PAL-1 protein (Draper et al., 1996; Hunter and Kenyon, 1996).

pal-1 encodes a Caudal-like homeodomain protein that is required to specify the fate of posterior blastomeres (Waring and Kenyon, 1990; Hunter and Kenyon, 1996). While pal-1 mRNA is present throughout the oocyte and early embryo, PAL-1 protein is not detected until the four-cell stage and then is only detected in posterior blastomeres (Hunter and Kenyon, 1996). This temporal and spatial patterning of PAL-1 expression is dependant on MEX-3, which contains two $\mathrm{KH}$ domain RNA binding motifs originally identified in hnRNP K (Draper et al., 1996; Siomi et al., 1994). mex-3 mutant hermaphrodites express PAL-1 in oocytes and all cells of their embryos, resulting in anterior-to-posterior homeotic transformations that are dependent on pal-1 (Hunter and Kenyon, 1996). These results suggest that the primary function of mex-3 in the early embryo is to repress PAL- 1 expression. Indeed, MEX-3 localization is complementary to that of PAL1. MEX-3 is present at high levels in mature oocytes, and oneand two-cell embryos, while at the four-cell stage, MEX-3 becomes enriched in anterior blastomeres relative to posterior blastomeres (Draper et al., 1996). Proper regulation of PAL-1 expression is also dependent on the pal-1 3' UTR, which can confer mex-3-dependent repression on reporter RNAs (Hunter and Kenyon, 1996). These observations suggest that MEX-3 may directly repress PAL-1 translation.

Not surprisingly, MEX-3 localization and activity is dependent on par activity. In par-1, par-4 and par-3 mutant four-cell embryos, MEX-3 is present at high levels in all cells, indicating that activity of these par genes is required to restrict MEX-3 to the anterior (Draper et al., 1996; Bowerman et al., 1997). In par- 1 and par-4 mutants, the presence of MEX-3 in all cells is coincident with failure to express PAL-1 in any cell, as would be expected if MEX-3 directly represses PAL-1 expression (Hunter and Kenyon, 1996; Bowerman et al., 1997). By contrast, four-cell par-3 embryos express PAL-1 in zero, two or four cells, despite uniformly high levels of MEX-3 in all cells, indicating that the mere presence of MEX-3 at the four-cell stage is not sufficient to repress PAL-1 expression (Bowerman et al., 1997). Finally, par-3 mutant embryos do not express PAL-1 before the four-cell stage, indicating that par-3 activity is required for spatial but not temporal control of PAL1 expression.

To explore further how MEX-3 translates the cell and embryonic polarity information provided by the par genes into the blastomere-specific pattern of PAL-1 expression, we analyzed par; mex-3 double mutants. Our results suggest that par activity promotes PAL-1 expression by inhibiting mex-3. To better understand how mex-3 activity is controlled, we identified and characterized two MEX-3 interacting proteins, MEX-6 and SPN-4, which are required to pattern PAL-1 expression. In addition to disrupting the pattern of PAL-1, inhibition of either gene by RNAi causes a unique terminal phenotype and has a distinct effect on MEX-3 expression levels. Investigation of genetic interactions between these genes and the par genes shows that par-1 and par-3 act upstream, providing polarity information that controls the spatial pattern of MEX-3 activity. By contrast, par-4 appears to act independently of par-1 and par-3 to inactivate MEX-3 at the four-cell stage. We conclude from these analyses that mex-3, mex-6 and spn-4 integrate spatial and temporal information from different sources to pattern PAL-1 expression in the early embryo.

\section{MATERIALS AND METHODS}

\section{Nematode strains and alleles}

Nematodes were cultured using standard conditions (Brenner, 1974). The Bristol strain N2 was used as the standard wild-type strain. The following mutant strains and alleles were also used: DP132, edIs6[unc119::GFP] IV; EU1, skn-1(zu67) IV/DnTl(IV;V); JJ462, +/nT1 IV; pos-1(zu148) unc-42(e270)/nT1 V; JJ1237, mex-6(pk440); JJ1238, unc-30(e191) mex-5(zu199) IV/nT1 (IV;V); JJ1244, mex6(pk440); unc-30(e191) mex-5(zu199) IV/nT1 (IV;V); KK184, par4(it47ts)V; KK288, rol-4(sc8) par-1(b274)IV/DnTl(IV;V); KK653, par-3(it71) unc-32(e189)/qC1 dpy-19(e1259ts) glp-1(q339) III; PD4251, ccIs4251[myo-3::GFP] I; dpy-20(e1282) IV; PD4790, mIs12[myo-2::GFP] ?; SU93, jcIs1 [jam-1::GFP] IV.

Embryos from homozygous mutant mothers are referred to as mutant embryos. All listed strains can be obtained from the Caenorhabditis Genetics Center, with the exception of KK653, which was kindly provided by Ken Kemphues.

\section{RNAi}

Sense and antisense single-stranded RNA was prepared from PCR products generated with primers that contained T7 and T3 RNA polymerase promoters and then annealed to produce double-stranded RNA (dsRNA). Hermaphrodites were injected with dsRNA and allowed to recover at $25^{\circ} \mathrm{C}$ for 16 or more hours before progeny were collected. Progeny from injected hermaphrodites are referred to as RNAi embryos. mex-3, mex-6 and spn-4 dsRNA was prepared from full-length cDNAs. par-3 dsRNA was prepared from a PCR product corresponding to bases 1743-2341 of the 4413 bp par-3-coding region. At the time embryos were collected for all experiments, $99 \%$ $(1539 / 1553)$ of mex-6(RNAi) embryos failed to hatch and $100 \%$ (1840/1843) of spn-4(RNAi) embryos failed to hatch.

\section{Yeast two-hybrid screen}

The yeast two-hybrid screen was conducted as described by Walhout and Vidal (Walhout and Vidal, 2001). Bases 10-634 of the 1248 bp mex-3-coding region, coding for half the protein and containing both $\mathrm{KH}$ domains, was fused to the gal-4 DNA-binding domain (DB) and used as bait in the screen. All constructs containing the $\mathrm{C}$ terminal half of MEX-3 fused to DB resulted in activation of reporter genes in the absence of any interacting protein, precluding its use in the screen. Approximately 200,000 yeast colonies were screened, yielding 14 unique positives. Library inserts were amplified from yeast as described (Walhout and Vidal, 2001) with the modification that T3 or T7 RNA polymerase sites were added to the ends of the oligonucleotides.

Inexplicably, injection of K07H8.10 dsRNA produced variable results from different preparations of RNA from identical templates. Three different preparations of RNA corresponding to three different regions of the predicted gene produced an RNAi phenotype characterized by embryonic lethality and PAL-1 expression in all cells starting at the four-cell stage. Numerous RNA preparations from the same templates using the same RNA synthesis protocol failed to produce embryonic lethality. Attempts to remedy the variability by using different regions of the RNA were unsuccessful. Because of these difficulties, we were unable to complete analysis of K07H8.10. 
We speculate that the variability between RNA preparations may be due to the extremely repetitive nature of the gene sequence, which may interfere with the formation of sufficiently long stretches of fully duplex double-stranded RNA.

\section{Terminal phenotype analysis}

Embryos fertilized 16 or more hours after injection were examined when uninjected control embryos had almost hatched. The following strains expressing tissue-specific GFP reporters (see nematode strains and alleles) were used to visualize the indicated tissue types: PD4251 (body wall muscle), SU93 (epidermis), PD4790 (pharyngeal muscle) and DP132 (neurons). Intestine was visualized by using polarizing optics to view intestinal cell-specific gut granules. Phenotypes were confirmed by differential interference contrast (DIC) microscopy of RNAi embryos from N2 worms. Representative embryos are shown but some variability in the terminal phenotype of $s p n-4(R N A i)$ embryos was observed. Specifically, spn-4(RNAi) embryos fertilized at later times after injection generally underwent less differentiation, most notably often failing to produce differentiated gut tissue.

\section{Antibodies and immunostaining}

PAL-1 immunostaining was performed as described (Hunter and Kenyon, 1996). Affinity purified anti-MEX-3 antibodies were prepared from the sera of rabbits immunized with full-length MEX$3::$ (his)6. Embryos were permeabilized by freeze-cracking and fixed by immersion in $-20^{\circ} \mathrm{C}$ methanol for 15 minutes, followed by $-20^{\circ} \mathrm{C}$ acetone for 15 minutes. Slides were rinsed in phosphate-buffered saline with $0.1 \%$ Tween (PBSt) before anti-MEX-3 antibodies were applied (1:100 in 3\% bovine serum albumin, Sigma A-9306). Slides were incubated overnight at $20^{\circ} \mathrm{C}$ and washed three times in PBSt before secondary antibody (Jackson Immunologicals) was applied.

\section{MEX-3 intensity measurements}

Optical sections of MEX-3-stained embryos were taken $1 \mu \mathrm{m}$ apart on the $z$ plane after a 0.4 second exposure using a DeltaVision microscope CCD camera (Applied Precision) and analyzed using softWoRx (Applied Precision) software. In no case was the camera saturated at this exposure setting. The center of each embryo was defined as the three contiguous sections with the highest Hoescht 33258 signal; all measurements were taken in all three center sections and then averaged. For each cell analyzed, raw MEX-3 intensity was measured from a circle of set diameter that contained the entire nucleus and that was fully enclosed by the cell membrane. A background measurement was taken a set distance away from the embryo in an area with no obvious cellular material and then subtracted from the raw MEX-3 intensity. Importantly, ABa and EMS lack MEX-3-containing P-granules in both wild-type and spn-4(RNAi) embryos, ensuring that we are only measuring cytoplasmic MEX-3. For comparison of $\mathrm{ABa}$ to EMS within the same type of embryo, measurements were taken for both cells from the same embryo. For comparison of spn-4(RNAi) cells with wild-type cells, an equal number of $s p n-4(R N A i)$ and wild-type embryos were analyzed from each experiment to minimize the effect of experiment to experiment variability. The two samples being compared were then subjected to Welch's approximate $t$-test, which is used to compare the means from two normal populations without assuming equal variances (Zar, 1999).

\section{RESULTS}

\section{par-1 and par-4 promote PAL-1 expression by inhibiting mex-3-mediated repression}

Four previously identified genes, mex-3, par-1, par-4 and par3 , are required to pattern PAL-1 expression (Hunter and Kenyon, 1996; Bowerman et al., 1997). To reveal aspects of the regulatory processes controlled by these proteins, we scored PAL-1 localization in oocytes and embryos from par; par and par; mex-3 double mutants that were created by injecting double-stranded RNA (dsRNA) specific to one gene into hermaphrodites mutant for a second gene (Fire et al., 1998). In all cases, injection of gene-specific dsRNA into wildtype adults produced a phenotype indistinguishable from that produced by strong loss-of-function alleles (data not shown). While highly penetrant RNAi and/or strong loss-of-function alleles were used for all experiments, there is a possibility that some residual activity remains.

We first examined the genetic relationship between par-1, par-4 and par-3 with respect to PAL-1 expression. par-3 mutants express PAL-1 in zero, two or four cells at the fourcell stage, while par-1 and par-4 mutants fail to express PAL1 in the early embryo (Fig. 1B-D, Fig. 2A) (Bowerman et al., 1997). We found that par-3(RNAi) embryos did not express
Fig. 1. PAL-1 immunolocalization in par and mex- 3 single and double mutant embryos. Strains mutant for the gene indicated above each column were injected with dsRNA as indicated to the left of each row (see Fig. 2A for quantitative summary of staining experiments). (A) Wild-type four-cell embryos express PAL-1 in the two posterior blastomeres. (B) par-3 embryos express PAL1 in variable patterns, including in all four blastomeres, as shown. (C) par-1 and (D) par4 embryos do not express PAL-1. (E-H) PAL1 is expressed in all blastomeres of all double mutants that include mex-3(RNAi). We observed that par-4; mex-3 embryos often failed to complete early cell cycle events normally, including nuclear division and cytokinesis. (I) par-1; par-3(RNAi) embryos and (J) par-4; par-3(RNAi) embryos do not express PAL-1. (K) Working model for control of PAL-1 expression. par-3 restricts par-1 and par-4 activities to the posterior, where they inhibit mex-3 repression of PAL-1. In this and all subsequent figures, embryos are oriented anterior left and dorsal upwards when it is possible to determine polarity. Scale bar: $10 \mu \mathrm{m}$.

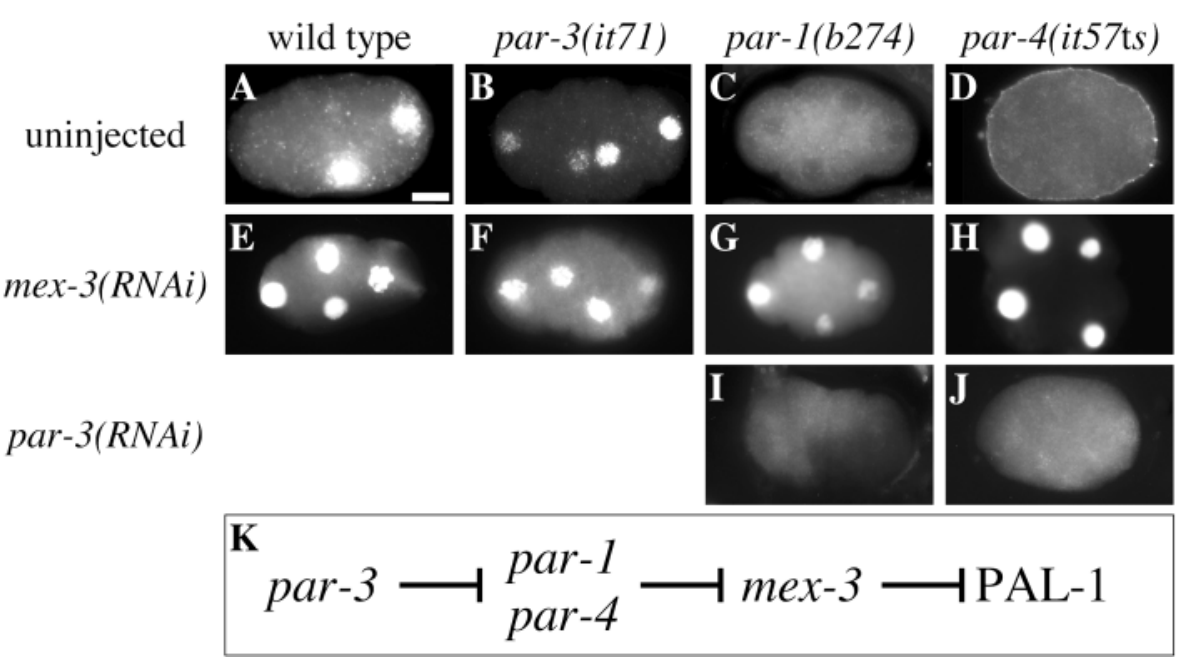




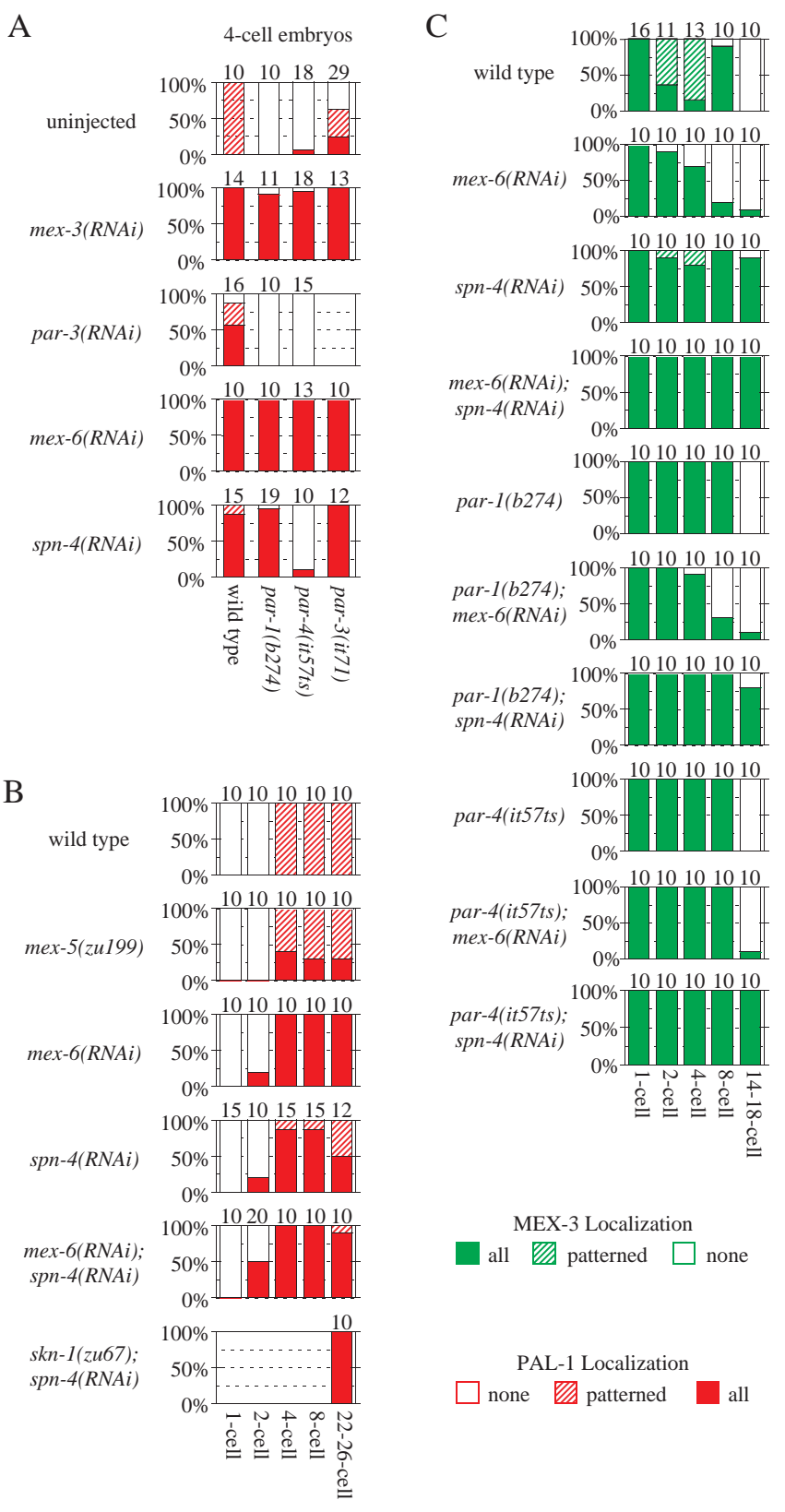

PAL-1 in the absence of par-1 or par-4 (Fig. 1I,J, Fig. 2A), suggesting that par-3 acts genetically upstream of par-1 and par-4 for control of PAL-1 expression. This is consistent with published results showing that anterior PAR-3 protein is required to restrict PAR-1 protein to the posterior cortex (Etemad-Moghadam et al., 1995) and may also explain the variable PAL-1 expression patterns observed in par-3(-) mutants, as par- 1 and par-4 activities may be variably distributed in par-3 mutant embryos.

As par- 1 and par- 4 are require for PAL-1 expression in wildtype and par-3(-) embryos, we next asked whether par-1 and par-4 are required for PAL-1 expression in mex-3 mutant embryos. In par-1; mex-3(RNAi) and par-4; mex-3(RNAi) double mutants, we found that PAL-1 was expressed at high levels in oocytes and all blastomeres (Fig. 1G,H, Fig. 2A), suggesting that par-1 and par-4 normally promote PAL-1 expression by inhibiting mex-3 activity. par-3; mex-3(RNAi) double mutants
Fig. 2. Quantitative summary of PAL-1 and MEX-3 localization data. (A) PAL-1 localization patterns in four-cell embryos. Wild-type, par1, par-4 or par-3 hermaphrodites, as indicated below the graphs, were injected with dsRNA, as indicated on the left. PAL-1 was scored as being present in none of the nuclei, all of the nuclei or patterned (see key). Patterned embryos express PAL-1 only in posterior blastomeres (see Fig. 1A), with the exception of par-3(it71) and par-3(RNAi) embryos, which express PAL-1 in two of four blastomeres but do not have an obvious polarity. Data for uninjected par-4(it57ts) and uninjected par-3(it71) embryos are taken from Bowerman et al. (Bowerman et al., 1997). (B) PAL-1 localization in embryos at different stages. Genotypes are indicated to the left and embryo stage is indicated below the graphs. In all cases in which PAL-1 was scored as patterned, PAL-1 was expressed only in posterior blastomeres (see Fig. 3B-D). (C) MEX-3 localization in wild-type and mutant embryos at different stages. Genotypes are indicated to the left and embryo stage is indicated below the graphs. In all two- and four-cell embryos in which MEX-3 was scored as patterned, MEX-3 was clearly brighter in the anterior blastomere(s) than in the posterior blastomere(s), as in the wild-type four-cell embryo in Fig. 5A2. In two- and four-cell embryos that were scored as 'all', MEX-3 was detected in all blastomeres with no clear asymmetries in signal intensity between anterior and posterior. Eightcell and 14- to 18-cell embryos were not scored for MEX-3 patterning and were scored only for the presence or absence of MEX-3 in the majority of blastomeres. In this way, wild-type embryos expressing MEX-3 in $\mathrm{P}_{2}$ blastomere descendants (see Fig. $5 \mathrm{~A} 4$ ) were scored as 'none' because they do not express MEX-3 in the majority of blastomeres. The data presented in these graphs reflect only the presence or absence of MEX-3 and do not reflect the intensity of MEX-3 signal, with the exception of clearly 'patterned' embryos, as described above. The total number of embryos scored is indicated above each bar in each case.

also express PAL-1 in the mex-3(-) pattern instead of in variable patterns (Fig. 1F, Fig. 2A). Similar results were obtained with mex-3; par-1 and mex-3; par-3 genetic double mutants (data not shown). A working model consistent with these results is presented in Fig. 1K; par-3 is upstream of par-1 and par-4, both of which are upstream of mex-3. It should be noted that this and all subsequent models are based on genetic data and are not intended to indicate direct physical interactions.

Consistent with the proposed model, at the two-cell stage, PAR-1 is enriched in the posterior blastomere that will give rise to all PAL-1-expressing cells (Guo and Kemphues, 1995). Two separate lines of evidence suggest that the spatial pattern of PAL1 expression is determined at the two-cell stage. First, PAL- 1 is detected in the posterior of all wild-type four-cell embryos (Fig. $2 \mathrm{~A}$ ), even if the posterior blastomeres have just completed cytokinesis. This indicates that PAL-1 synthesis begins immediately after division, suggesting that the regulatory machinery that promotes PAL-1 expression is in place before division. Second, four-cell par-3(-) embryos express PAL-1 in zero, two or four cells, never one or three cells (Fig. 2A) (Bowerman et al., 1997). The two anterior cells always express PAL-1 at the same level, and the two posterior cells always express PAL-1 at the same level. These observations suggest that the decision to express PAL-1 is made independently in each blastomere at the two-cell stage, even though the result of this decision is not evident until the four-cell stage.

MEX-3 interacting proteins regulate PAL-1 expression The spatial pattern of MEX-3 localization correlates with the 
Table 1. MEX-3 two-hybrid screen results

\begin{tabular}{|c|c|c|c|c|}
\hline $\begin{array}{l}\text { Number of } \\
\text { isolates }\end{array}$ & Gene & Similarity & Terminal phenotype* & PAL- $1^{\dagger}$ \\
\hline 4 & mex-3 & Two KH domains & Embryonic arrest & All \\
\hline 1 & mex- 6 & Two CCCH type zinc fingers & Embryonic arrest & All starting at the four-cell stage \\
\hline 1 & K07H8.10 & RNA recognition motif & Undifferentiated cells & All starting at the four-cell stage \\
\hline 2 & T04D 1.3 & GRB2-like SH3 domain & Embryonic arrest & Variable abnormal \\
\hline 20 & pos-1 & Two CCCH type zinc fingers & Embryonic arrest & Variable abnormal \\
\hline 1 & B0250.1 & Ribosomal protein L8 & Undifferentiated cells & Variable abnormal \\
\hline 1 & C43E11.9 & RNA-binding domain & Undifferentiated cells & Wild type \\
\hline 1 & F40F8.5 & Novel & Viable, fertile & $\mathrm{n} / \mathrm{d}$ \\
\hline 1 & K03B4.7 & Novel & Viable, fertile & $\mathrm{n} / \mathrm{d}$ \\
\hline 1 & C27H5.3 & RNA recognition motif & Viable, fertile & $\mathrm{n} / \mathrm{d}$ \\
\hline 1 & E02D9.1 & Protein kinase domain & Viable, fertile & $\mathrm{n} / \mathrm{d}$ \\
\hline
\end{tabular}

*Phenotypes of embryos resulting from RNAi (see Materials and Methods). All embryos produced differentiated tissue, except as noted.

${ }^{\dagger}$ PAL-1 staining in young embryos.

\# K07H8.10(RNAi) showed inconsistent effects from different preparations of dsRNA (see Materials and Methods) and was therefore only minimally analyzed. $\mathrm{n} / \mathrm{d}$, not determined.

pattern of PAL-1 repression in wild-type, par-1 and par-4 embryos (Draper et al., 1996; Bowerman et al., 1997). However, par-3 mutants express PAL-1 in variable patterns at the four-cell stage, despite uniformly high levels of MEX-3 in all cells, indicating that MEX-3 is not sufficient to repress PAL-1 at this stage (Bowerman et al., 1997). To identify additional proteins that regulate PAL-1 expression, we conducted a yeast two-hybrid screen with the N-terminal half of MEX-3 as bait. The screen of 200,000 transformants yielded 14 unique MEX-3 interacting proteins or MIPs (Table 1). Many of these MIPs contain sequence similarity to factors implicated in RNA metabolism, consistent with the putative role of MEX-3 as an RNA-binding protein. We then used RNAi to determine whether any of the identified MIPs are required to regulate PAL-1 expression. Depletion of three of the MIPs, mex-6, ZC404.8 and K07H8.10, caused PAL-1 to be expressed in all blastomeres starting at the four-cell stage (Fig. 2B, Fig. 3E-L and data not shown), while depletion of the other MIPs resulted in wild-type or variable PAL-1 expression (Table 1). K07H8.10 (RNAi) showed inconsistent effects from different preparations of dsRNA (see Materials and Methods) and was therefore only minimally analyzed. Our analysis of mex- 6 and ZC404.8 is described in the following sections.

\section{mex -5 and $m e x-6$ are required for spatial, but not temporal repression of PAL-1}

Before being identified as a MIP, mex- 6 was characterized because it shares high sequence identity with $m e x-5$, a maternal effect lethal gene that causes cell fate transformations leading to excess muscle when defective (Schubert et al., 2000). mex5 and mex- 6 encode $70 \%$ identical proteins with two $\mathrm{CCCH}$ type zinc fingers, motifs also found in the maternal proteins PIE-1, MEX-1 and POS-1 (Mello et al., 1996; Guedes and Priess, 1997; Tabara et al., 1999). Work by Schubert and colleagues indicates that mex-5 and mex-6 have overlapping functions; mex-6 single mutants are viable, but mex-5; mex-6 double mutants have a more severe phenotype than mex-5 single mutants. We find that RNAi with full-length mex-6 results in ectopic PAL-1 in anterior cells at high penetrance, a phenotype not observed in mex-5 or mex-6 single mutants (Fig.
Fig. 3. PAL-1 immunolocalization in wildtype, mex-6(RNAi) and spn-4(RNAi) embryos. Genotypes and embryo stages are indicated (see Fig. 2B for quantitative summary of staining experiments). (A-D) Wild-type embryos express PAL-1 only in posterior blastomeres starting at the four-cell stage. (E-H) mex-6(RNAi) embryos express PAL-1 at equally high levels in all blastomeres starting at the four-cell stage. (F) In the four-cell mex6(RNAi) embryo shown, the two anterior cells are dividing before the posterior cells, demonstrating that mex-6(RNAi) embryos can maintain asymmetries in cell cycle time through the four-cell stage. For all 22- to 26cell embryos, some nuclei are out of the plane of focus. (I-L) spn-4(RNAi) embryos express PAL-1 in all blastomeres at the four-cell stage; PAL-1 is often detected at lower levels in anterior blastomeres than posterior blastomeres, as evident in the eight-cell embryo shown (K). Approximately half of (L) spn-4(RNAi) 22- to 26-cell embryos express PAL-1 in all blastomeres as shown, the remainder express PAL-1 only in posterior blastomeres, similar to (D) wild-type embryos (see Fig. 2B for quantitation). spn-4(RNAi) embryos characteristically maintain asymmetries in cell cycle time as evident in the (K) eight-cell embryo shown, where the anterior blastomeres have proceeded to prophase while the posterior blastomeres are still in interphase. 
Fig. 4. Terminal phenotypes and cell type abundance in mip(RNAi) embryos. The DIC micrographs in the top row show that mex-3, mex- 6 and spn-4 RNAi embryos produce differentiated cell types but do not undergo

morphogenesis. In the remaining rows, body wall muscle, epidermis, intestine, pharynx and neurons were visualized as described in Materials and Methods. mex-3, mex-6 and spn-4 RNAi embryos produce excess body wall muscle and epidermal cells. mex-3 and spn-4 RNAi embryos produce a normal amount of intestine but reduced pharynx, while mex-6(RNAi) embryos produce no intestine and excess pharynx. By the time the neural GFP marker was expressed, neural tissue was dispersed throughout the embryo, making it difficult to judge the amount of neural tissue. Thus, we conclude only that neural tissue is present in mex-3, mex-6 and spn-4 RNAi embryos and do not make any conclusions about the relative amount. For all embryos, some

fluorescent signal is out of the plane of focus. This is most noticeable in the intestine micrographs, where the wildtype intestine has elongated but the mex3 and spn-4 RNAi intestine has not.
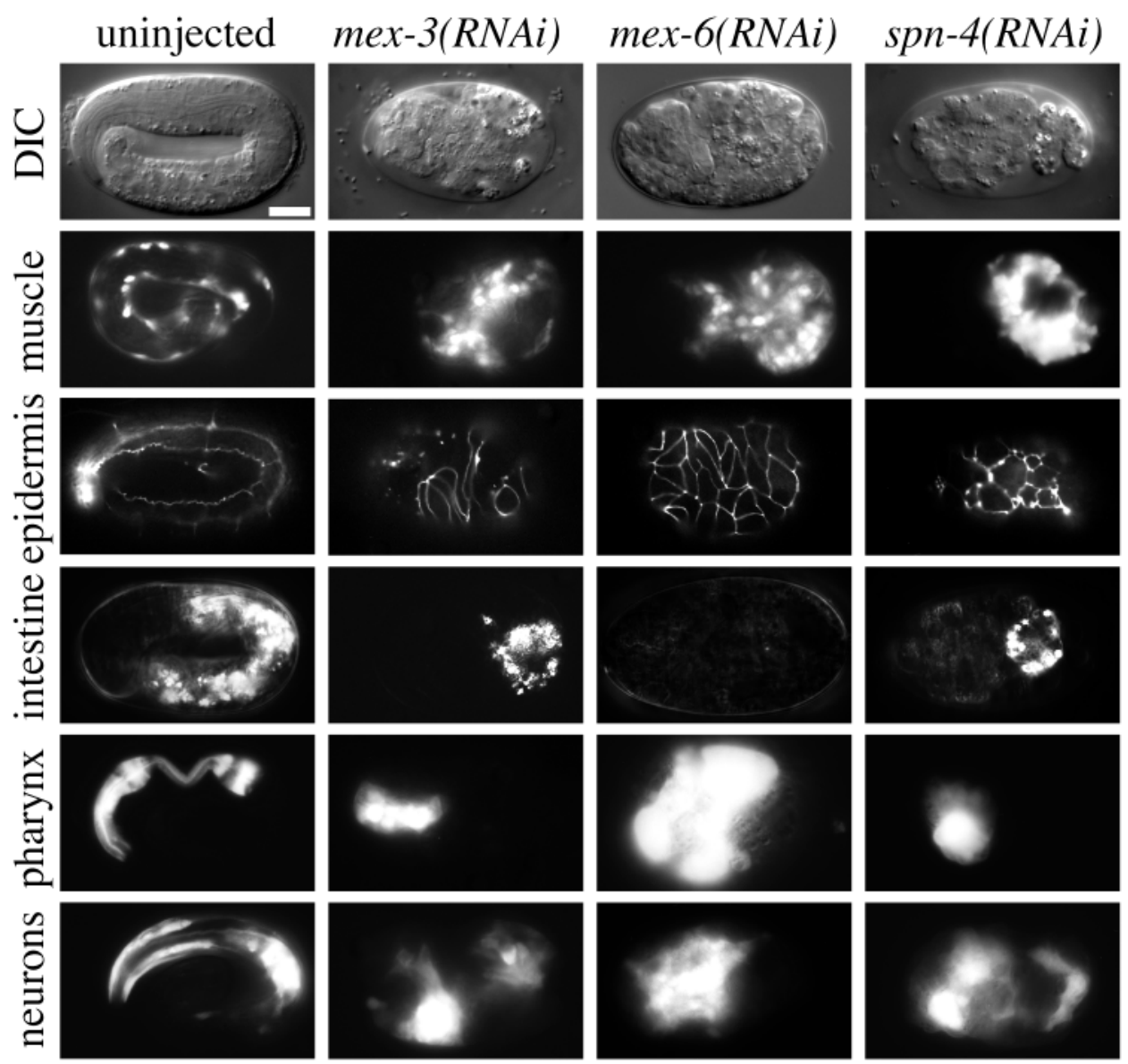

2B; data not shown). These results suggest that injecting fulllength mex- 6 dsRNA reduces the function of both mex-5 and mex-6. Indeed, these genes share extensive regions of nucleotide identity, including seven regions of 30 nucleotides or longer with greater than $90 \%$ nucleotide identity, conditions that exceed the minimal requirements for effective genespecific RNAi (Parrish et al., 2000). Therefore, we interpret our results obtained with full-length mex- 6 dsRNA as reducing the function of both mex-5 and mex-6.

mex-6(RNAi) embryos, like mex-3(-) embryos, failed to undergo morphogenesis and produced excess muscle and epidermal cells (Fig. 4). However, mex-6(RNAi) embryos also contained an excess of pharyngeal cells and completely lacked gut cells, while mex-3 mutant embryos produce a reduced number of pharyngeal cells and a normal amount of gut. Like mex-6(RNAi) embryos, the genetic mex-5(zu199) mutant produces an excess of pharyngeal cells. Curiously, the mex6(pk440); mex-5(zu199) double mutant produces fewer pharyngeal cells (Schubert et al., 2000). These results indicate that mex-6(RNAi) may only partially inhibit mex-5 and that residual mex-5 or mex- 6 activity may promote pharyngeal development. With the exception of pharynx production, the mex-6(RNAi) phenotype appears to be identical to the mex6(pk440); mex-5(zu199) double mutant phenotype (data not shown).

mex-5 and mex- 6 were previously shown to be required for the posterior localization of a number of maternal proteins, including PAL-1 (Schubert et al., 2000). To determine whether mex-5 and mex- 6 are required for the temporal control of PAL1 expression, like mex-3, or the spatial control of PAL-1 expression, like par-3, we stained mex-6(RNAi) embryos for PAL-1. We found that mex-6(RNAi) disrupts spatial but not temporal control of PAL-1 expression, resulting in embryos that show strong PAL-1 expression in all nuclei starting at the four-cell stage (Fig. 2B, Fig. 3E-H). The ectopic PAL-1 expression in mex-6(RNAi) embryos is consistent with the excess muscle and excess epidermis observed, which are fates promoted by the homeodomain protein PAL-1.

\section{spn-4 is required for spatial, but not temporal repression of PAL-1}

ZC404.8 encodes an RNA recognition motif (RRM) protein that was also identified as a mutant with defects in spindle rotation, spn-4, and as a POS-1 interacting protein, pip-1 (Gomes et al., 2001) (K. Ogura and Y. Kohara, personal communication). pos-1, which was also identified in our twohybrid screen with MEX-3, encodes a germline-restricted protein containing two $\mathrm{CCCH}$ zinc-finger motifs also found in mex-5 and mex-6 (Tabara et al., 1999). spn-4(RNAi) produced mex-3-like embryos that failed to undergo morphogenesis, produced excess muscle, excess epidermal cells, a reduced number of pharyngeal cells and a normal amount of intestine (Fig. 4) (at later times after injection, an increasing proportion of animals fail to differentiate intestine and other tissues - see Materials and Methods).

Starting at the four-cell stage, spn-4(RNAi) embryos 
consistently showed weak ectopic PAL-1 expression in anterior cells in addition to wild-type PAL-1 expression in the posterior (Fig. 2B, Fig. 3I-L). (See Fig. 3K to compare weak versus strong staining in an eight-cell embryo.) This indicates that spn-4, like mex-5 and mex-6 and par-3, is required for spatial, but not temporal patterning of PAL-1. The ectopic PAL-1 expression in spn-4(RNAi) embryos is consistent with the mex3-like terminal phenotype.

\section{mex-5, mex-6 and spn-4 have opposite effects on MEX-3 expression levels}

To determine whether mex-5, mex-6 and spn-4 affect MEX-3 expression or activity, we stained mex-6(RNAi) and spn4(RNAi) embryos with MEX-3 antibodies. In mex-6(RNAi) oocytes and newly fertilized one-cell embryos, MEX-3 was distributed throughout the cell at levels similar to wild type (Fig. 2C and data not shown). However, by the four-cell stage, MEX-3 levels in all blastomeres were lower than is typically seen in wild-type posterior blastomeres (Fig. 5A2,B2). Furthermore, MEX-3 was undetectable by the eight-cell stage, while it is readily detectable throughout wild-type eight-cell embryos (Fig. 2C, Fig. 5A3,B3). These results suggest that the anteriorly localized MEX-5 and MEX-6 proteins (Schubert et al., 2000) (N. N. H. and C. P. H., unpublished) are required to stabilize MEX-3 in the anterior to pattern its expression. If MEX-3 is required at the four-cell stage to repress PAL-1 expression, the ectopic PAL-1 in mex-6(RNAi) embryos can be explained solely by the failure to stabilize MEX-3, although we cannot rule out the possibility that mex-5 and mex- 6 may be directly involved in PAL-1 repression.

By contrast, in young spn-4(RNAi) embryos, MEX-3 protein levels appeared higher in all blastomeres than is ever detected in wild type, and in most cases was not clearly patterned (Fig. $2 \mathrm{C}$, Fig. 5C). This indicates that MEX-3 is either not active or is insufficient at the four-cell stage to repress PAL-1 expression in spn-4(RNAi) embryos. Furthermore, we found that MEX-3 staining persisted much longer than in wild type. In 14- to 18cell wild-type embryos, MEX-3 is undetectable except for weak staining in the $\mathrm{P}_{2}$ blastomere descendants (Fig. 5A4), whereas in spn-4(RNAi) embryos, MEX-3 is readily detectable at the 14- to 18-cell stage and in some cases even persists through the 20- to 30-cell stage (Fig. 2C, Fig. 5C4 and data not shown). These results suggest that spn-4 is required to inhibit MEX-3 accumulation in older embryos.

To determine whether the presence of MEX-3 at the 14- to 18-cell stage actually reflects an overabundance of MEX-3 at the four-cell stage, when PAL-1 expression is perturbed, we used image analysis software to measure the intensity of MEX3 staining in wild-type and spn-4(RNAi) four-cell embryos (Fig. 6A-C). We measured MEX-3 intensity in the anterior blastomere $\mathrm{ABa}$, which normally expresses high levels of MEX-3, and in the posterior blastomere EMS, which normally expresses low levels of MEX-3. In wild-type embryos, we found that $\mathrm{ABa}$ was always brighter than EMS (Fig. 6A), reflecting the qualitative differences observed by eye. By contrast, much smaller differences were found in spn-4(RNAi) embryos between $\mathrm{ABa}$ and EMS, demonstrating that spn-4 is required to pattern MEX-3 (Fig. 6B).

To determine whether MEX-3 levels were higher in spn$4(R N A i)$ embryos than in wild type, we compared the measured levels between embryos. Although there is considerable variation in the antibody staining, a statistically significant difference was observed between spn-4(RNAi) and wild-type $\mathrm{ABa}$ blastomeres (Fig. 6C), indicating that spn-4(RNAi) embryos do contain an overabundance of MEX-3 at the fourcell stage. The largest difference was observed between spn-4(RNAi) and wild-type EMS blastomeres (Fig. 6C), underscoring both the lack of patterning in spn-4(RNAi) embryos and the higher overall levels of MEX-3 in spn$4(R N A i)$ embryos. These observations strongly suggest that spn-4 acts to limit MEX-3 levels and that its activity is preferentially targeted to the posterior blastomeres of four-cell embryos.

These quantitative measurements reflect what we qualitatively observe at the four-cell stage. Furthermore, these data suggest that the persistence of MEX-3 at the 14- to 18cell stage in spn-4(RNAi) embryos does reflect an overabundance of MEX-3 at the four-cell stage. Because of the difficulty in making these measurements, and the relatively large sample sizes required to obtain statistically significant comparisons, all remaining samples were assayed solely for the presence or absence of MEX-3 at the 8- and 14- to 18-cell stages. For all genotypes, the persistence of MEX-3 appeared to correlate with the staining intensity in young embryos.

\section{mex-5 and mex-6 prevent spn-4-dependent degradation of MEX-3}

Although mex-5, mex- 6 and spn-4 are required to repress PAL1 expression in anterior blastomeres, they have opposite effects on MEX-3 expression levels. To determine whether mex-5 and mex- 6 are required for the abnormal accumulation of MEX-3 observed in spn-4(RNAi) embryos, or whether spn-4 is required for the abnormally low levels of MEX-3 observed in mex6(RNAi) embryos, we stained mex-6(RNAi); spn-4(RNAi) double mutant embryos for MEX-3. In the double mutants, MEX-3 persisted through the 14- to 18-cell stage, as in spn4(RNAi) embryos (Fig. 2C, Fig. 5D), suggesting that anterior mex-5 and mex-6 (Schubert et al., 2000) (N. N. H. and C. P. H., unpublished) normally act to restrict spn-4-dependent degradation of MEX-3 to the posterior. As an indication that the mex- 6 dsRNA was active in these co-injection experiments, MEX-3-stained P-granules were distributed throughout the double mutant embryos as in mex-6(RNAi) embryos (see Fig. 5D2).

Despite the persistent MEX-3 staining observed in spn$4(R N A i)$ embryos, PAL-1 is expressed at high levels in the posterior blastomeres and at low levels in the anterior blastomeres. To determine whether mex-5 and mex- 6 are required for this anterior-posterior asymmetry in PAL-1 expression, we stained mex-6(RNAi); spn-4(RNAi) double mutant embryos for PAL-1. In these double mutants, PAL-1 was expressed strongly in all blastomeres (Fig. 2B and data not shown), suggesting that mex-5 and mex-6 partially inhibit PAL-1 expression in the anterior of spn-4(RNAi) embryos. Consistent with this interpretation, we found that MEX-5 was localized normally to the anterior of two-cell and four-cell spn4(RNAi) embryos (data not shown).

From these results we are able to place mex-5, mex-6 and spn-4 into a working model for regulation of PAL-1 expression (Fig. 6D). spn-4 acts to promote MEX-3 degradation while mex-5 and mex- 6 normally act in the anterior to stabilize MEX-3 and allow it to repress PAL-1. 
Fig. 5. MEX-3

immunolocalization in wild-type and mutant embryos. Genotypes and embryo stages are indicated (see Fig. 2C for quantitative summary of staining experiments). (A) In wildtype embryos, MEX-3 is present through the eightcell stage and essentially disappears by the 14- to 18 cell stage, except for weak staining in the $\mathrm{P}_{2}$

blastomere descendants.

(B) In mex-6(RNAi)

embryos, MEX-3 is

undetectable by the eight-

cell stage. (C,D) In spn-

4(RNAi) embryos and mex6(RNAi); spn-4(RNAi)

embryos, MEX-3 persists

through to the 14- to 18-cell stage. (E) In par-1 embryos, MEX-3 persists through the eight-cell stage but is undetectable by the 14- to 18-cell stage. (F,G) Defects in par-1 do not effect the MEX-3 phenotype of mex6(RNAi) embryos or spn4(RNAi) embryos. (H) In par-4 embryos, MEX-3 persists through the eightcell stage but is undetectable by the 14- to 18 -cell stage. (I) par-4 is required for the premature degradation of MEX-3 seen in mex-6(RNAi) embryos.

(J) Defects in par-4 do not affect the MEX-3

phenotype of spn-4(RNAi) embryos. Slight variations in the intensity of MEX-3 signal in the micrographs are often the result of the embryo position in the focal plane, and may not reflect actual asymmetries in protein distribution. Only clear asymmetries that are visible in all focal planes, as in A2, were scored as patterned (see Fig. 2C).

Representative embryos are

shown. Owing to the high intensity of the signal, $(\mathrm{C}, \mathrm{D}, \mathrm{G}, \mathrm{J}) \mathrm{spn}-4(\mathrm{RNA} i)$ two- and four-cell embryos are generally underexposed relative to other embryos. All eight-cell embryos are shown at the same exposure, with the exception of $\mathrm{C} 3$ and G3, which are underexposed relative to the other eight-cell embryos. All 14- to 18-cell embryos are shown at the same exposure with the exception of C4, G4 and J4, which are underexposed relative to the other 14- to 18-cell embryos. In two-cell (B1) mex-6(RNAi) and (C1) spn-4(RNAi) embryos, the anterior cell is typically larger than the posterior cell as in (A1) wild type, demonstrating that mex- 6 and spn-4 RNAi embryos can maintain asymmetries in cell size. mex-6(RNAi) disrupts P-granule localization as monitored by MEX-3 localization to P-granules (Draper et al., 1996). Ten out of 10 mex-6(RNAi) embryos mis-segregate P-granules to all blastomeres of the four-cell embryo, while 10/10 of spn-4(RNAi) embryos segregate P-granules normally. (D) mex-6(RNAi); spn-4(RNAi) double mutant embryos show persistent MEX-3 expression characteristic of spn-4(RNAi) embryos, while 8/10 four-cell embryos mislocalize P-granules like mex-6(RNAi) embryos, indicating that both injected dsRNAs were effective in RNAi. 

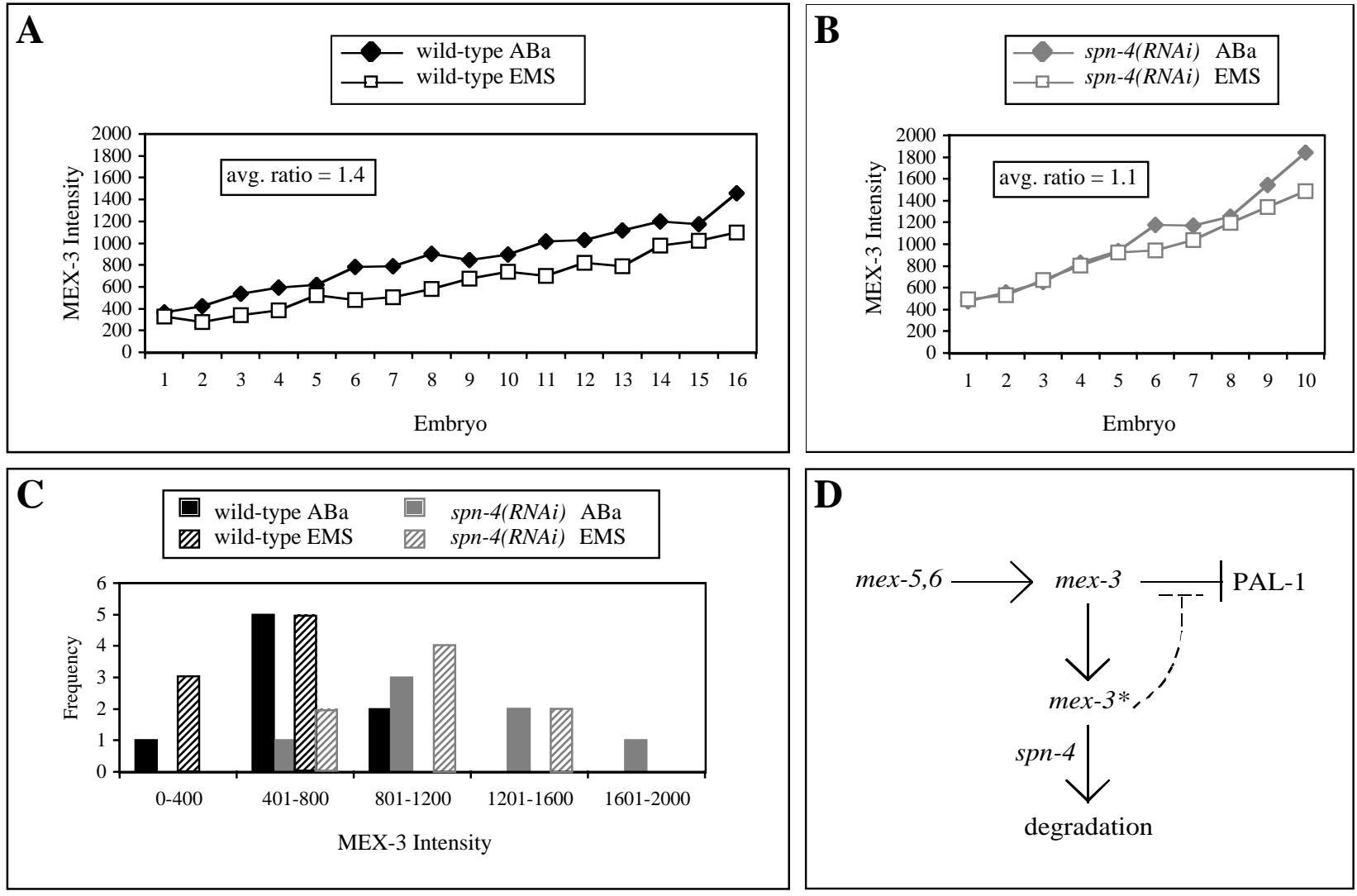

\begin{tabular}{|c|c|c|c|}
\hline Cell & Mean \pm s.d. (n) & Ratio & t-test \\
\hline$\frac{s p n-4(R N A i)}{\text { wild-type ABa }} \mathrm{ABa}$ & $\frac{1101 \pm 443(8)}{645 \pm 220(8)}$ & 1.7 & $\mathrm{P}<0.05$ \\
\hline$\frac{s p n-4(R N A i)}{\text { wild-type EMS }} \frac{\mathrm{EMS}}{\frac{985 \pm 331(8)}{477 \pm 127(8)}}$ & 2.1 & $\mathrm{P}<0.005$ \\
\hline
\end{tabular}

Fig. 6. MEX-3 intensity and working model. MEX-3 intensity in $\mathrm{ABa}$ and EMS from individual (A) wild-type or (B) spn-4(RNAi) embryos. The average ratio between $\mathrm{ABa}$ and EMS intensities is given. (C) Comparison of MEX-3 intensity in wild-type and spn-4(RNAi) blastomeres. Units are arbitrary for MEX-3 intensity. (D) Working model for regulation of PAL-1 expression. MEX-3 exists in active and inactive (*) forms. The active form is normally associated with mex-5 and mex-6 and is competent to repress PAL-1. In the absence of mex-5 and mex- 6 , the inactive form is targeted for degradation by spn-4. In the absence of spn-4, the inactive MEX-3 accumulates and interferes with active MEX-3.

Based on the observation that MEX-3 is present but inactive in par-3 and spn-4(RNAi) embryos, we suggest that mex-3 exists in active and inactive forms. The active form is normally associated with mex-5 and mex- 6 and is competent to repress PAL-1 while the inactive form is normally targeted for degradation by $s p n-4$. In the absence of $s p n-4$, we propose that inactive MEX-3 accumulates and interferes with active MEX3 , resulting in the weak ectopic PAL-1 expression seen in spn4(RNAi) embryos.

\section{par-3 and par-1 act upstream of mex-5, mex- 6 and spn-4}

To determine how mex-5, mex- 6 and spn-4 interact with the par genes that are known to affect PAL-1 expression, we immunostained mip; par double mutants for PAL-1. par-3 embryos express PAL-1 in variable patterns starting at the four-cell stage, while par-1 and par-4 embryos fail to express PAL-1 at all in the early embryo (Fig. 2A, Fig. 7A-C). By contrast, mex-6(RNAi) and spn-4(RNAi) embryos express PAL-1 in all cells at the four-cell stage (Fig. 2A, Fig. 3F,J). We found that PAL-1 was expressed in all mip; par-3 and mip; par-1 double mutants (Fig. 2A, Fig. 7D,E,G,H), suggesting that par-3 and par- 1 function upstream of mex-5, mex- 6 and spn-4 to regulate PAL-1 expression. This is consistent with published results showing that par-1 is required to localize MEX-5 to the anterior while PAR-1 and PAR-3 are localized normally in mex-5(-); mex-6(-) embryos (Schubert et al., 2000) and the results indicating that par-3 is upstream of par1. Furthermore, the characteristic par-3 cell arrangement (evident in Fig. 7A,D) was often (9/10) suppressed by spn$4(R N A i)$ in par-3; spn-4(RNAi) embryos (Fig. 7G), again consistent with spn-4 acting downstream of par-3. Two-cell par-3(-) embryos characteristically divide with both spindles oriented anteroposteriorly (Cheng et al., 1995), while two-cell spn-4 embryos characteristically divide with both spindles oriented dorsoventrally (Gomes et al., 2001).

MEX-3 localization in mip; par-1 embryos is also consistent with par-1 acting upstream of mex-5, mex-6 and spn-4. par-1 is not required for the premature degradation of MEX-3 seen in mex-6(RNAi) embryos (Fig. 2C, Fig. 5F) or for the excess accumulation of MEX-3 seen in spn-4(RNAi) embryos (Fig. 2C, Fig. 5G). This suggests that mex-5, mex-6 and spn-4 work downstream of or parallel to par-1 with respect to MEX-3 localization. These data allow us to integrate par-1, par-3, mex- 
Fig. 7. PAL-1 immunolocalization in par; mip(RNAi) double mutant embryos. Strains mutant for the gene indicated above each column were injected with dsRNA (left) (see Fig. 2A for quantitative summary of staining experiments). (A) par-3 mutants express PAL-1 in variable patterns, including in all four blastomeres, as shown. (B,C) par-1 and par-4 mutants do not express PAL-1. (D-F) All double mutants that include mex6(RNAi) express PAL-1 at a high level in all four blastomeres. $(\mathrm{G}, \mathrm{H})$ par-3; spn-4(RNAi) embryos and par-1; spn-4(RNAi) embryos express PAL-1 in all four blastomeres. The characteristic par-3 cell arrangement (evident in A,D) was suppressed by spn$4(R N A i)$ in $9 / 10(\mathrm{G})$ par-3; spn-4(RNAi) embryos. (I) par-4; spn4(RNAi) embryos do not express PAL-1. We observed that par-4; spn-4(RNAi) embryos, like par-4; mex-3(RNAi) embryos (see Fig. 1), often failed to complete early cell cycle events normally, including nuclear division and cytokinesis.

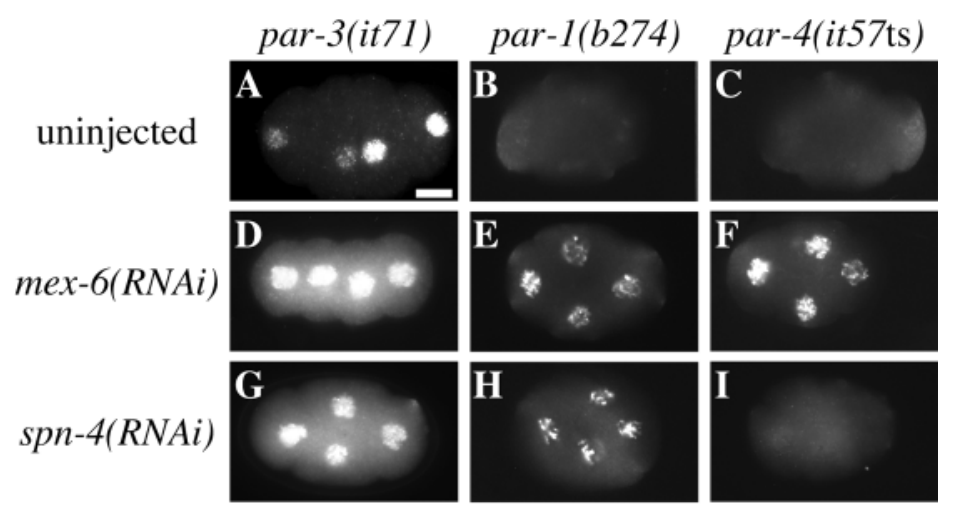

5, mes-6 and spn-4 into one working model for the regulation of PAL-1 expression (Fig. 8B). Anteriorly localized par-3 restricts par- 1 to the posterior. Posterior par- 1 then restricts mex-5 and mex- 6 to the anterior, where they stabilize MEX-3 and allow it to repress PAL- 1 expression in the anterior. In the absence of mex-5 and mex-6 in the posterior, MEX-3 is degraded through a spn-4-dependent process.

\section{par-4 analysis indicates that spn-4 acts in a separate pathway from mex-5 and mex- 6 to pattern PAL-1}

We found that par-4 activity is not required for PAL-1 expression in mex-6(RNAi) embryos (Fig. 2A, Fig. 7F), but par-4 is required for PAL-1 expression in spn-4(RNAi) embryos (Fig. 2A, Fig. 7I), suggesting that spn-4 acts in a separate pathway from mex-5 and mex-6 to control PAL-1 expression. We next looked at MEX-3 expression in mip: par4 double mutant embryos. We found that par-4 is not required for the abnormal persistence of MEX-3 seen in spn-4(RNAi) embryos (Fig. 2C, Fig. 5J), but par-4 is required for the premature degradation of MEX-3 seen in mex-6(RNAi) embryos (Fig. 2C, Fig. 5I). We showed earlier that this premature degradation is also dependent on spn-4 activity, indicating that par-4 and spn-4 are both required for MEX-3 degradation in mex-6(RNAi) embryos. Furthermore, par-4 is required for PAL-1 expression in spn-4(RNAi) embryos (Fig. 2A, Fig. 7I), suggesting that par-4 inactivates MEX-3 or a MEX-3 co-factor. We suggest in our working model in Fig. 8B that par-4 is required to inactivate MEX-3 and target it for spn4-dependent degradation.

\section{spn-4 is required to restrict skn-1 expression and function}

spn-4(RNAi) embryos differ from both mex-3(-) and mex6(RNAi) embryos in an important respect; in a large proportion of spn-4(RNAi) embryos, PAL-1 expression regresses towards a wild-type pattern. This is particularly evident at the 22- to 26-cell stage when zygotic pal-1 transcription is thought to begin (Fig. 2B). In wild-type 22to 26-cell embryos, PAL-1 expression begins to decrease in the EMS descendants relative to the $\mathrm{P}_{2}$ descendants (Hunter and Kenyon, 1996). In homozygous pal-1 null embryos from heterozygous mothers, PAL-1 expression in the $\mathrm{P}_{2}$ descendants begins decreasing at this time as well, indicating that the continued high expression of PAL-1 in the $\mathrm{P}_{2}$ descendants requires zygotic pal- 1 transcription (Edgar et al., 2001; Hunter and Kenyon, 1996).

Interestingly, PAL-1 zygotic expression appears to require pal-1 function. The transcription factor SKN-1 inhibits pal-1 function in the EMS lineage (Bowerman et al., 1993; Hunter and Kenyon, 1996). In 22- to 26-cell skn-1 mutants, PAL-1 levels remain high in the EMS descendants (C. P. H., unpublished). Thus, the observation that half the spn-4(RNAi) embryos expressed PAL-1 in wild-type patterns at the time of maternal to zygotic transition in PAL-1 expression suggests that the ectopic PAL-1 may not be active in half the embryos.

A

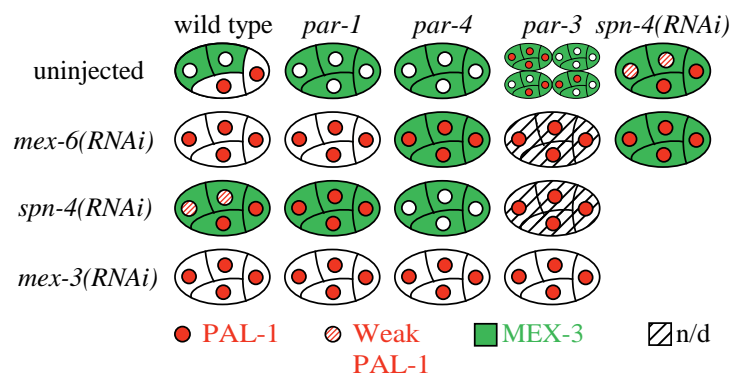

B

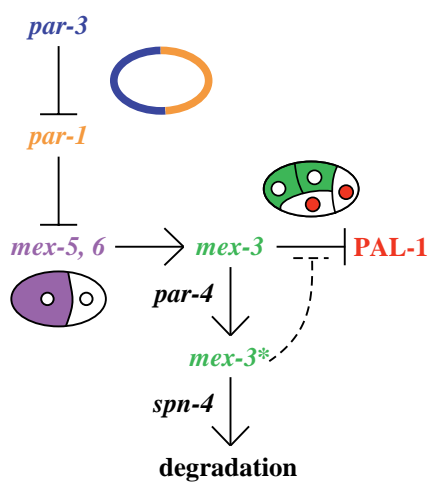

Fig. 8. Summary and proposed model.

(A) Summary of PAL-1 (red) and MEX-3 (green) localization data. (B) Working model for regulation of PAL-1 expression. Anteriorly localized PAR-3 (blue) restricts cortical PAR1 (orange) to the posterior. par- 1 then restricts MEX-5 and MEX-6 (purple) to the anterior. mex-5 and mex- 6 protect MEX-3 from degradation in the anterior, enabling the continued

repression of PAL-1. In the absence of mex-5 and mex- 6 in the posterior, par-4 inactivates MEX-3 (*), and subjects it to rapid spn-4dependent degradation. In the absence of $s p n-4$, inactive MEX-3 can interfere with active MEX-3, resulting in ectopic PAL-1 expression. 
One possibility is that SKN-1 may be anteriorly expressed in some spn-4(RNAi) embryos and may inhibit the activation of zygotic pal-1 expression. To test this, we first stained spn4(RNAi) embryos for SKN-1. We found four-cell embryos that expressed SKN-1 predominantly in the two posterior blastomeres, as in wild type (Bowerman et al., 1993), as well as embryos that expressed SKN-1 strongly in all four blastomeres (data not shown). If this anterior SKN-1 can inhibit zygotic PAL-1 expression, then PAL-1 should continue to be expressed in the anterior blastomeres of all $s k n-1$; spn$4(R N A i)$ embryos. Therefore, we injected skn-1 mutant hermaphrodites with spn-4 dsRNA and found that, as predicted, all double mutant embryos continued to express PAL-1 in anterior blastomeres through the 22- to 26-cell stage (Fig. 2B). These experiments show that spn-4 is required to pattern SKN-1 localization and that ectopic SKN-1 inhibits at least some pal-1 functions.

\section{DISCUSSION}

We have identified two proteins, MEX-6 and SPN-4, which are required to pattern PAL-1 expression at the four-cell stage. Our characterization of their genetic interactions with the other factors known to affect PAL-1 patterning, mex-3, par-1, par-4 and par-3, suggests that these two genes couple the spatial and temporal information provided by the par genes to the spatial and temporal pattern of gene expression. Previous work has shown that mex-3 is required to repress PAL- 1 expression and that MEX-3 localization in oocytes and early embryos correlates with the temporal and spatial pattern of PAL-1 repression (Hunter and Kenyon, 1996; Draper et al., 1996). Although the striking correlation between MEX-3 localization and PAL-1 repression is suggestive, in some mutants PAL-1 is expressed in blastomeres that contain high levels of MEX-3 (Bowerman et al., 1997), indicating that MEX-3 activity is regulated independently of expression levels.

Our analysis of par; mex-3(RNAi) double mutants suggests that the patterned expression of PAL-1 at the four-cell stage requires PAL-1 repression in oocytes and in one- and two-cell embryos. If PAL-1 is expressed in the oocyte, as it is in mex3 mutants, all blastomeres will inherit PAL-1 protein and translationally active pal-1 RNA, thereby disrupting the spatial regulation of PAL-1 expression. Furthermore, analysis of par; par-3(RNAi) double mutants indicates that both par-1 and par4 are required to promote PAL-1 expression at the four-cell stage when mex-3-dependent repression is intact. These results also show that par-3 mutations do not disrupt the regulatory interactions that pattern PAL-1, just their spatial distributions in two-cell embryos. To clarify these interactions and to gain further understanding of how cell polarity information can produce asymmetric patterns of gene expression, we sought to identify additional genes required to pattern PAL-1 expression. As MEX-3 is necessary but insufficient to repress PAL-1 expression, we used a two-hybrid screen to identify MEX-3 interacting proteins and then used RNAi to characterize their functions. By these methods we identified two proteins, SPN4 and MEX-6, which produced a consistent and informative RNAi phenotype suitable for detailed investigations.

Previous analysis of mex-5 and mex- 6 indicates that these homologous proteins function redundantly and act downstream of par- 1 to control the asymmetric expression and/or activity of a variety of factors important for early embryogenesis (Schubert et al., 2000). Among these, PAL-1 is the only one for which a specific regulator has been identified, namely MEX-3. We found that mex-5 and mex- 6 are required for the continued expression of MEX-3 in early embryos and also for continued repression of PAL- 1 in anterior blastomeres at the four-cell stage. For both these phenotypes, mex-6(RNAi) is epistatic to par-1(-), consistent with the requirement for par1 in localizing MEX-5 to the anterior blastomere of two-cell embryos (Schubert et al., 2000). Thus, it appears that MEX-5 and MEX-6 act as adapters to transmit the polarity information conferred by the par genes into the control of PAL-1 expression. As MEX-6 was identified as a MEX-3 interacting protein, one possibility is that MEX-5 and MEX-6 act as corepressors with MEX-3 at the four-cell stage. Indeed, all double mutants that included mex-6(RNAi) express PAL-1 in all blastomeres of four-cell embryos whether MEX-3 is present or not (summarized in Fig. 8A).

A direct interaction between MEX-3 and anteriorlylocalized MEX-5 and MEX-6 may also provide a mechanism for maintaining MEX-3 levels in anterior blastomeres. A physical interaction between MEX-5, MEX-6 and MEX-3, or some modification resulting from a physical interaction, could protect MEX-3 from protein degradation. Nevertheless, MEX5 and MEX-6 are not absolutely required for MEX-3 stability, as MEX-3 is stable in par-4(-) and spn-4(RNAi) embryos, whether or not mex-5 and mex- 6 are active. This suggests that par-4(+) and spn-4(+) may be required to destabilize MEX-3.

In all genetic backgrounds tested, reducing the function of par-4 or spn-4 resulted in elevated MEX-3 levels in the posterior, indicating that par-4 and spn-4 may normally be required to reduce $\mathrm{MEX}-3$ levels in the posterior. Because PAR-4 and SPN-4 are localized to all cells of two- and fourcell embryos (Watts et al., 2000) (K. Ogura and Y. Kohara, personal communication; N. N. H. and C. P. H., unpublished), their activity must be asymmetrically controlled in the anterior and posterior blastomeres. This difference could be attributed to MEX-5 and MEX-6, which are localized to the anterior and may act to protect MEX-3 from par-4- and spn-4-dependent degradation.

Despite these similarities, par-4 and spn-4 have different effects on PAL-1 expression. In spn-4(RNAi) embryos, weak ectopic PAL-1 was detected in anterior blastomeres and normal levels of PAL-1 were detected in posterior blastomeres. Therefore, the abnormally abundant MEX-3 in spn-4(RNAi) embryos is either not active or insufficient to repress PAL-1 expression. However, this PAL-1 expression is completely dependent on par-4 activity. Thus, as in wild-type and par-3(-) embryos, par-4 activity is required to promote PAL-1 expression in spn-4(RNAi) embryos. One hypothesis consistent with all the data is that par-4 activity directly or indirectly inactivates MEX-3. The observation that par-1 and par-4 mutations have different effects on PAL-1 expression in spn4(RNAi) embryos provides evidence that par-1 and par-4 act by distinct mechanisms to pattern PAL-1 expression at the four-cell stage.

Because PAL-1 expression is dependent on both par-1 and par-4, one attractive hypothesis is that one of these serine/threonine kinases (PAR-1) spatially restricts PAL-1 derepression to the posterior, and the other (PAR-4) temporally 
derepresses PAL-1 expression after the two-cell stage. At the two-cell stage, cortically localized PAR-1 is restricted to the posterior blastomere that will give rise to all the PAL-1expressing cells (Guo and Kemphues, 1995). By contrast, PAR-4 is uniformly localized to both blastomeres at the twocell stage, suggesting it may be active in temporal regulation (Watts et al., 2000). par-4 is unique among the par genes in that it is not asymmetrically localized and does not affect asymmetry of the first cleavage (Kemphues et al., 1988), suggesting that it may act later than par-1 and par-3, perhaps providing a temporal cue for early embryonic events including PAL-1 derepression. In this scenario, all blastomeres in fourcell par-1 mutant embryos execute the fate of anterior blastomeres that do not derepress PAL-1, and all blastomeres in four-cell par-4 mutant embryos maintain the state of younger blastomeres that do not yet express PAL-1. As par-3 activity is required for PAR-1 localization (Etemad-Moghadam et al., 1995), par-3 mutants may variably distribute par-1 activity, resulting in the variable PAL-1 expression patterns that are observed.

Precedence for separable temporal and spatial control of post-transcriptional gene regulation in C. elegans is provided by the analysis of GLP-1 (Evans et al., 1994). $g l p-1$ mRNA is present throughout the oocyte and early embryo, while GLP-1 protein is expressed only in anterior blastomeres from the twoto 28-cell stage. cis regulatory elements have been mapped to two distinct regions in the $g l p-13^{\prime} \mathrm{UTR}$, with one region being required for temporal control and the other for spatial patterning. As the pal-1 3'UTR can confer a PAL-1-like expression pattern on reporter RNAs (Hunter and Kenyon, 1996), PAL-1 expression may be regulated by RNA-binding proteins such as MEX-3 and the MIPs bound to the 3'UTR. In turn, temporal and spatial activity of MEX-3 and the MIPs would ultimately be controlled by the par genes.

These complex genetic interactions are synthesized into a formal genetic model in Fig. 8B in which separate temporal and spatial control pathways converge on MEX-3 to control its activity and stability at the four-cell stage. First, we propose that par-3 and par-1 function in a simple linear pathway to localize MEX-5 and MEX-6 to the anterior (EtemadMoghadam et al., 1995; Schubert et al., 2000) (N. N. H. and C. P. H., unpublished), where they function to stabilize and/or activate MEX-3. This stabilization probably involves direct contact as we identified MEX-6 as a MIP. In addition to stabilizing MEX-3, mex-5 and mex- 6 are required at the fourcell stage for the continued repression of PAL-1. This is demonstrated by the par-4; mex-6(RNAi) and mex-6(RNAi); spn-4(RNAi) embryos, which express PAL-1 at high levels in all blastomeres despite abundant MEX-3.

Temporal control of PAL-1 expression may be provided by par-4(+) activity. We propose that par-4 acts directly or indirectly to inactivate MEX-3-dependent repression of PAL1 at the two- to four-cell stage. The inactivated MEX-3 is then subject to rapid spn-4-dependent degradation. mex-5 and mex6 activity in the anterior protects MEX-3 from inactivation and subsequent degradation, thus restricting PAL-1 expression to the posterior. To explain the weak PAL-1 expression in the anterior of spn-4(RNAi) embryos, we propose that inactive MEX-3 interferes with active MEX-3. The identification of MEX-3 as a MEX-3 interacting protein (Table 1) is consistent with MEX-3 forming an oligomer. However, we must emphasize that the interactions between MEX-3 and the MIPs were identified in yeast and may not reflect physical interactions in the $C$. elegans embryo.

As par-4 encodes a serine/threonine kinase, it is tempting to speculate that it may inactivate MEX-3 by directly phosphorylating it. Phosphorylation of $\mathrm{KH}$ domain proteins has been shown to affect their RNA binding activity (Wang et al., 1995). Furthermore, phosphorylation is often used to target proteins for degradation (for a review, see Jackson et al., 2000). Finally, MEX-3 is hyperphosphorylated and rapidly degraded in wild-type early embryos (C. P. H., unpublished).

As MEX-3, MEX-5, MEX-6 and SPN-4 all contain RNAbinding motifs, an appealing hypothesis is that these proteins interact on the pal-1 3'UTR. However, mex-3 is the only factor that appears to be specific for regulation of PAL-1 expression; mex-5 and mex- 6 are required to segregate several unrelated gene products, and spn-4 is required to control spindle orientation and SKN-1 localization (Draper et al., 1996; Hunter and Kenyon, 1996; Schubert et al., 2000; Gomes et al., 2001) (our data). This raises the possibility of combinatorial control, where MEX-5, MEX-6 and SPN-4 may each act on numerous RNA targets to control their expression, perhaps by regulating the activity and stability of other message-specific trans-acting factors similar to MEX3 . The concept of combinatorial control is well documented in transcriptional control, where individual transcription factors can act as activators or repressors of transcription depending on the other factors that are bound (for a review, see Roberts, 2000). Combinatorial control is also emerging as a dominant theme in translational control, but few specific examples are well understood (for a review, see Gray and Wickens, 1998).

Asymmetric protein expression resulting from posttranscriptional regulation is best understood in Drosophila. However, Drosophila embryos are distinct from nematode and vertebrate embryos because they are polarized before fertilization and begin development as a syncytial blastoderm. This means that maternal RNAs and proteins can be localized during oocyte maturation and that after fertilization, RNA and protein can diffuse freely through the cytoplasm. Perhaps because of these unique characteristics, many factors important for converting polarity cues to asymmetric gene expression during early Drosophila development do not have homologs in other systems; two notable exceptions being Caudal/PAL-1 (Macdonald and Struhl, 1986; Waring and Kenyon, 1991) and Par-1 (Guo and Kemphues, 1995; Shulman et al., 2000). In the C. elegans embryo, many asymmetrically expressed proteins have been identified, but relatively little is known about how that asymmetric expression is achieved (for a review, see Goodwin and Evans, 1997). The new findings presented in this work provide a framework for understanding how initial cellular polarity can be refined to produce asymmetric gene expression in a cellular system.

We thank J. Gomes, S. E. Encalada, K. A. Swan, C. A. Shelton, J. C. Carter and B. Bowerman for communicating work before publication. We also thank K. Ogura and Y. Kohara for communicating work before publication. We are grateful to $\mathrm{C}$. Schubert and J. Priess for providing anti-MEX-5 antibodies and mex5 and mex-6 mutant strains. We thank J. M. Rihel and A. J. Kay for thoughtful review of this manuscript. This work was supported in part by a March of Dimes Basil O'Connor scholar award (C. P. H.), 
National Institutes of Health grant GM 61677 (C. P. H.) and National Institutes of Health grant R01HG01715A1 (M. V.). N. N. H. is partially supported by an NSF predoctoral fellowship.

\section{REFERENCES}

Bowerman, B. (1998). Maternal control of pattern formation in early Caenorhabditis elegans embryos. Curr. Top. Dev. Biol. 39, 73-117.

Bowerman, B., Draper, B. W., Mello, C. C. and Priess, J. R. (1993). The maternal gene $s k n-1$ encodes a protein that is distributed unequally in early C. elegans embryos. Cell 74, 443-452.

Bowerman, B., Ingram, M. K. and Hunter, C. P. (1997). The maternal par genes and the segregation of cell fate specification activities in early Caenorhabditis elegans embryos. Development 124, 3815-3826.

Brenner, S. (1974). The genetics of Caenorhabditis elegans. Genetics 77, 7194.

Cheng, N. N., Kirby, C. M. and Kemphues, K. J. (1995). Control of cleavage spindle orientation in C. elegans: the role of the genes par-2 and par-3. Genetics 139, 549-559.

Draper, B. W., Mello, C. C., Bowerman, B., Hardin, J. and Priess, J. R. (1996). MEX-3 is a KH domain protein that regulates blastomere identity in early C. elegans embryos. Cell 87, 205-216.

Edgar, L. G., Carr, S., Wang, H. and Wood, W. B. (2001). Zygotic Expression of the caudal Homolog pal-1 Is Required for Posterior Patterning in Caenorhabditis elegans Embryogenesis. Dev. Biol. 229, 7188.

Etemad-Moghadam, B., Guo, S. and Kemphues, K. J. (1995). Asymmetrically distributed PAR-3 protein contributes to cell polarity and spindle alignment in early C. elegans embryos. Cell 83, 743-752.

Evans, T. C., Crittenden, S. L., Kodoyianni, V. and Kimble, J. (1994). Translational control of maternal glp-1 mRNA establishes an asymmetry in the C. elegans embryo. Cell 77, 183-194.

Fire, A., Xu, S., Montgomery, M. K., Kostas, S. A., Driver, S. E. and Mello, C. C. (1998). Potent and specific genetic interference by double-stranded RNA in Caenorhabditis elegans. Nature 391, 806-811.

Goldstein, B. and Hird, S. N. (1996). Specification of the anteroposterior axis in Caenorhabditis elegans. Development 122, 1467-1474.

Gomes, J. E., Encalada, S. E., Swan, K. A., Shelton, C. A., Carter, J. C. and Bowerman, B. (2001). The maternal gene spn-4 encodes a predicted RRM protein required for mitotic spindle orientation and cell fate patterning in early C. elegans embryos. Development 128, 4301-4314.

Goodwin, E. B. and Evans, T. C. (1997). Translational control of development in C. elegans. Semin. Cell Dev. Biol. 8, 551-559.

Gray, N. K. and Wickens, M. (1998). Control of translation initiation in animals. Annu. Rev. Cell Dev. Biol. 14, 399-458.

Guedes, S. and Priess, J. R. (1997). The C. elegans MEX-1 protein is present in germline blastomeres and is a $\mathrm{P}$ granule component. Development 124, 731-739.

Guo, S. and Kemphues, K. J. (1995). par-1, a gene required for establishing polarity in $C$. elegans embryos, encodes a putative Ser/Thr kinase that is asymmetrically distributed. Cell 81, 611-620.

Hung, T. J. and Kemphues, K. J. (1999). PAR-6 is a conserved PDZ domaincontaining protein that colocalizes with PAR-3 in Caenorhabditis elegans embryos. Development 126, 127-135.

Hunter, C. P. and Kenyon, C. (1996). Spatial and temporal controls target pal-1 blastomere-specification activity to a single blastomere lineage in $C$. elegans embryos. Cell 87, 217-226.

Jackson, P. K., Eldridge, A. G., Freed, E., Furstenthal, L., Hsu, J. Y., Kaiser, B. K. and Reimann, J. D. R. (2000). The lore of the RINGS: substrate recognition and catalysis by ubiquitin ligases. Trends Cell Biol. 10, 429-439.

Joberty, G., Petersen, C., Gao, L. and Macara, I. G. (2000). The cellpolarity protein Par6 links Par3 and atypical protein kinase C to Cdc42. Nat. Cell Biol. 2, 531-539.

Kemphues, K. J., Priess, J. R., Morton, D. G. and Cheng, N. S. (1988), Identification of genes required for cytoplasmic localization in early $C$. elegans embryos. Cell 52, 311-320.

Kemphues, K. J. and Strome, S. (1997). Fertilization and establishment of polarity in the embryo. In C. elegans II (ed. D. L. Riddle), pp.335-359. Plainview, NY: Cold Spring Harbor Laboratory Press.

Lin, D., Edwards, A. S., Fawcett, J. P., Mbamalu, G., Scott, J. D. and Pawson, T. (2000). A mammalian PAR-3-PAR-6 complex implicated in Cdc42/Rac1 and aPKC signalling and cell polarity. Nat. Cell Biol. 2, 540-547.

Macdonald, P. M. and Struhl, G. (1986). A molecular gradient in early Drosophila embryos and its role in specifying the body pattern. Nature 324, 537-545.

Mello, C. C., Schubert, C., Draper, B., Zhang, W., Lobel, R. and Priess, J. R. (1996). The PIE-1 protein and germline specification in C. elegans embryos. Nature 382, 710-712.

Parrish, S., Fleenor, J., Xu, S., Mello, C. and Fire, A. (2000). Functional anatomy of a dsRNA trigger: Differential requirement for the two trigger strands in RNA interference. Mol. Cell 6, 1077-1087.

Roberts, G. G. E. (2000). Mechanisms of action of transcription activation and repression domains. Cell. Mol. Life Sci. 57, 1149-1160.

Schubert, C. M., Lin, R., de Vries, C. J., Plasterk, R. H. and Priess, J. R. (2000). MEX-5 and MEX-6 function to establish soma/germline asymmetry in early C. elegans embryos. Mol. Cell 5, 671-682.

Shulman, J. M., Benton, R. and St Johnston, D. (2000). The Drosophila homolog of C. elegans PAR-1 organizes the oocyte cytoskeleton and directs oskar mRNA localization to the posterior pole. Cell 101, 377-388.

Siomi, H., Choi, M., Siomi, M. C., Nussbaum, R. L. and Dreyfuss, G. (1994). Essential role for KH domains in RNA binding: impaired RNA binding by a mutation in the KH domain of FMR1 that causes fragile $\mathrm{X}$ syndrome. Cell 77, 33-39.

Tabara, H., Hill, R. J., Mello, C. C., Priess, J. R. and Kohara, Y. (1999). pos-1 encodes a cytoplasmic zinc-finger protein essential for germline specification in C. elegans. Development 126, 1-11.

Walhout, A. J. and Vidal, M. (2001). High-throughput yeast two-hybrid assays for large-scale protein interaction mapping. Methods 24, 297-306.

Wang, L. L., Richard, S. and Shaw, A. S. (1995). P62 association with RNA is regulated by tyrosine phosphorylation. J. Biol. Chem. 270, 2010-2013.

Waring, D. A. and Kenyon, C. (1990). Selective silencing of cell communication influences anteroposterior pattern formation in C. elegans. Cell 60, 123-131.

Waring, D. A. and Kenyon, C. (1991). Regulation of cellular responsiveness to inductive signals in the developing $C$. elegans nervous system. Nature 350, $712-715$.

Watts, J. L., Morton, D. G., Bestman, J. and Kemphues, K. J. (2000). The C. elegans par- 4 gene encodes a putative serine-threonine kinase required for establishing embryonic asymmetry. Development 127, 1467-1475.

Zar, J. J. (1999). Biostatistical Analysis. Upper Saddle River, NJ: Prentice Hall. 